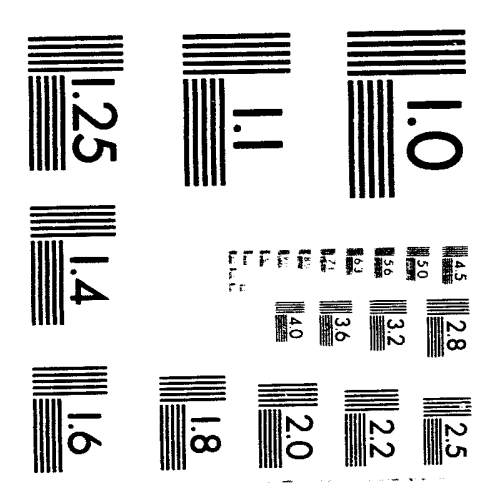



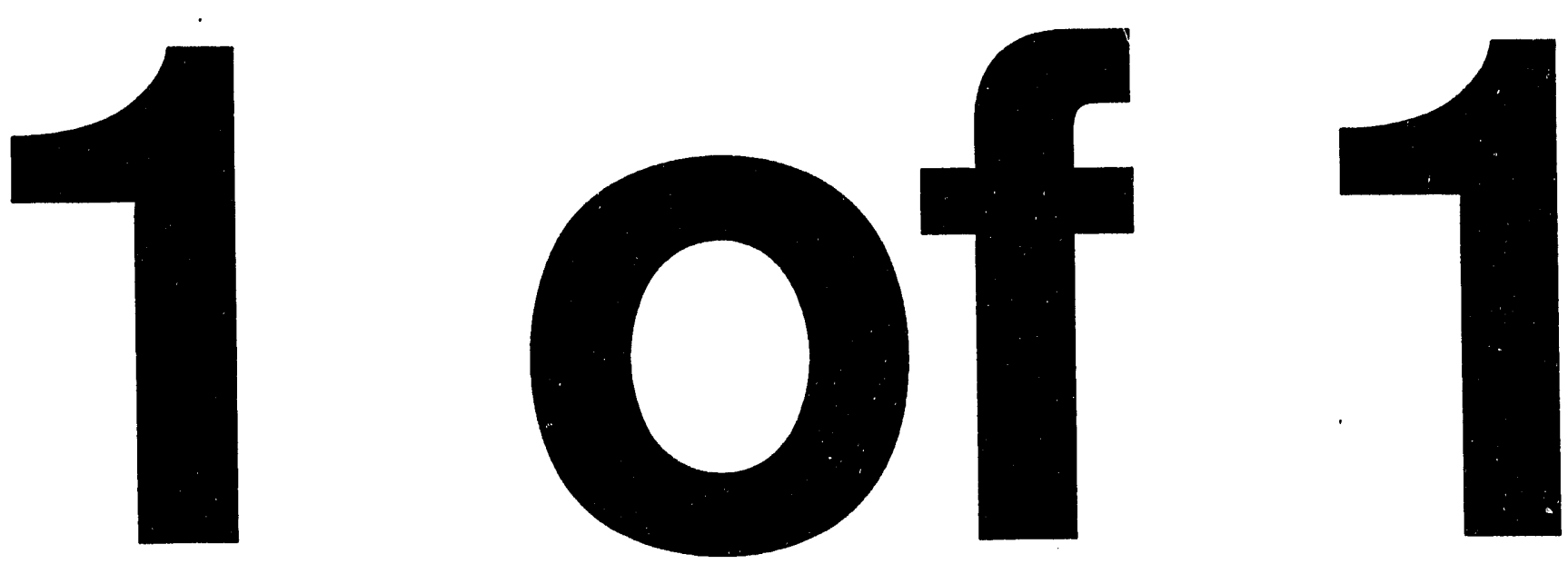


\title{
Conf-9309173-.9
}

UCRL JC- 113392

PREPRINT

\section{Survey of Odd-Odd Deformed Nuclear Spectroscopy}

\author{
Richard W. Hoff
}

This report was presented as an invited paper at the Eighth International Symposium on Capture Gamma-Ray Spectroscopy and Related Topics, Fribourg, Switzerland, September 20-24, 1993

September 14, 1993

$=$ This is a preprint of a paper intended for publication in a journal or proceedings. Since changes may be made before publication, this preprint is made available with the understanding that it will not be cited or reproduced without the permission of the author. 


\section{DISCLAIMER}

This document was prepared as an account of work sponsored by an agency of the United States Government. Neither the United States Government nor the University of California nor any of their employees, makes any warranty; express or implied, or assumes any legal liability or responsibility for the accuracy, completeness, or usefulness of any information, apparatus, product, or process disclosed, or represents that its use would not infringe privately owned rights. Reference herein to any specific commercial products, process, or service by trade name, trademark, manufacturer. or otherwise, does not necessarily constitute or imply its endorsement, recommendation, or favoring by the United States Government or the University of California. The views and opinions of authors expressed herein do not necessarily state or reflect those of the United States Government or the University of California, and shall not be used for advertising or product endorsement purposes. 


\title{
SURVEY OF ODD-ODD DEFORMED NUCLEAR SPECTROSCOPY*
}

\author{
RICHARD W. HOFF \\ Lawrence Livermore National Laboratory \\ Livermore, California 94550, USA
}

\begin{abstract}
In this paper, we survey the current experimental data that support assignment of rotational bands in odd-odd deformed nuclei in the rare earth and actinide regions. We present the results of a new study of ${ }^{170} \mathrm{Tm}$ nuclear structure. In a comparing experimental and calculated Gallagher-Moszkowski matrix elements for rare earth-region nuclei, we have developed a new approach to the systematics of these matrix elements.
\end{abstract}

\section{Introduction}

The complex level structure and decay schemes encountered in the study of oddodd deformed nuclei present many challenges to both experimentalists and theorists. These nuclei display all of the phenomena encountered in other deformed nuclei, collective motion due to rotation and vibration, quasiparticle excitations, etc., plus a unique phenomenon, the interaction between two unpaired and unlike nucleons which can be observed at low excitation energies. Since the odd-odd nucleus has the potential for a great variety of combinations of different quasiparticle excitations, even at low energies, the level densities observed are higher than those seen in neighboring nuclei. Thus, it is essential that oddodd nuclei be probed using spectroscopic techniques than can provide highest resolution and highest sensitivity. With the advent of modern high-flux research reactors, neutroncapture gamma-ray spectroscopy as performed at centers such as the Institut Laue-Langevin (ILL) and the Brookhaven National Laboratory (BNL) has become the single most important experimental probe for examining the nuclear structure of odd-odd deformed nuclei at low levels of angular momentum. Another very important technique, one that is essential to any comprehensive study of low-energy nuclear structure, is the use of singlenucleon transfer reactions with a modern high-resolution charged-particle spectrometer as exemplified by studies performed at the Technical University of Munich's (TUM) tandem accelerator.

\section{Survey of Experimental Data}

The current status of spectroscopic studies of certain quadrupole deformed nuclei in the rare earth and actinide regions is summarized in Table 1. Included in the table are those odd-odd nuclei that can be formed in neutron-capture reactions with stable isotope targets plus a few additional nuclides $\left({ }^{164} \mathrm{Ho},{ }^{168} \mathrm{Tm}\right.$, and $\left.{ }^{174} \mathrm{Lu}\right)$ whose low-lying nuclear 
structures have been determined experimentally in considerable detail. This summary provides details on the observation of secondary transitions and assignment of their multipolarities, the identification of excited levels and their assignment to rotational bands, and the number of $n-p$ interaction matrix elements derived from the rotational band data. Due to limitations on space, references to the relevant experimental papers for the data summarized in Table 1 are not given. A summary of all rotational bands determined experimentally in odd-odd deformed nuclei ${ }^{1}$ will be published toward the end of 1993.

One can judge whether the present-day experimental facilities for thermal neutroncapture spectroscopy have been exploited to their full potential by referring to some of the data in Table 1. For example, the values in column 4, numbers of secondary transitions observed (in the energy range $0-1500 \mathrm{keV}$ ) per barn of thermal neutron-capture cross section, show that for ${ }^{160} \mathrm{~Tb},{ }^{176} \mathrm{Lu},{ }^{182} \mathrm{Ta}$, and ${ }^{244} \mathrm{Am}$, this ratio takes on values $\geq 10$, presumably indicating the results of exhaustive and sensitive searches for secondary transitions. For ${ }^{152} \mathrm{Eu}$ and ${ }^{154} \mathrm{Eu}$, which are formed by thermal capture reactions that have large cross sections, the numbers of secondary transitions are reported up to maximum energies of only 510 and $550 \mathrm{keV}$, respectively. Apparently, the density of lines in their spectra above these energies is so great as to make further analysis difficult or impossible. Certain of the heavier rare earth-region nuclei such as ${ }^{166} \mathrm{Ho},{ }^{170} \mathrm{Tm},{ }^{186} \mathrm{Re}$, and ${ }^{188} \mathrm{Re}$ have moderately large cross sections and might very well yield appreciably more data on secondary transitions if further measurements are made with the GAMS spectrometers following restart of the reactor at the ILL. In the actinide region, only ${ }^{244} \mathrm{Am}$ appears to have been exhaustively studied. However, the heat generated by neutron-induced fission provides a limitation to increasing the mass of actinide targets that does not apply in the case of rare earth targets. Although the odd-mass actinide target isotopes themselves are not very fissionable, the odd-odd products of neutron capture are highly fissionable. Nevertheless, certain targets, e.g. ${ }^{237} \mathrm{~Np}$, appear to be good candidates for a repeat of the gamma-ray measurements using GAMS 1,2/3.

We can see from column 8 of Table 1 that the conversion electron spectra of ${ }^{160} \mathrm{~Tb}$, ${ }^{166} \mathrm{Ho},{ }^{182} \mathrm{Ta}$, and ${ }^{188} \mathrm{Re}$ have not been studied with a sensitive beta spectrometer. For each of these nuclei, less than $15 \%$ of the observed secondary transitions have multipolarities assigned from conversion electron measurements. These measurements should be made, but they will be more difficult to accomplish now that the BILL spectrometer at the ILL has been permanently dismantled. Fortunately, all actinide nuclei in Table 1 have had their conversion electron spectra measured with the BILL spectrometer except ${ }^{228} \mathrm{Ac}$, where problems of handling a highly radioactive target would make the measurement extremely challenging.

The number of levels given in column 9 of Table 1 is that from all experimental measurements. On the basis of rotational bands assigned, ${ }^{152} \mathrm{Eu},{ }^{154} \mathrm{Eu}$, and ${ }^{176} \mathrm{Lu}$ appear to be the most extensively characterized, while $160 \mathrm{~Tb}$ and ${ }^{186} \mathrm{Re}$, of the nuclei populated by neutron capture, seem to offer the greatest opportunities for further study. For the nuclei shown in the table, a total of $99 \mathrm{E}(\mathrm{GM})$ and $34 \mathrm{E}(\mathrm{N})$ experimental matrix elements have been derived. 
Table 1. Status of odd-odd deformed nuclear spectroscopy

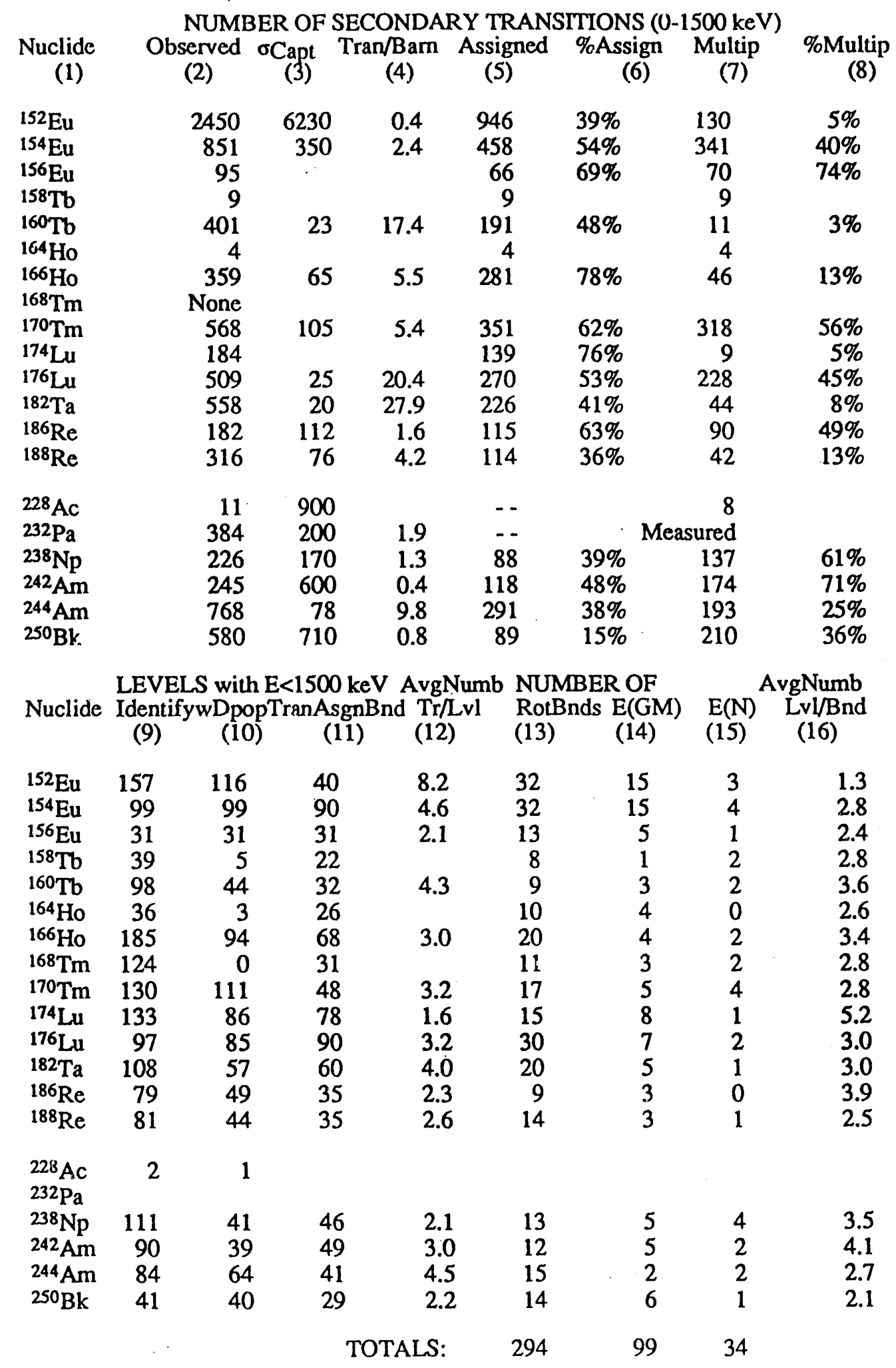




\section{Measurements of the ${ }^{170} \mathrm{Tm}$ Decay Scheme}

In the present investigation, the following experimental quantities were determined: Energies and intensities of secondary gamma rays and conversion electrons from measurements of the thermal-neutron ${ }^{169} \mathrm{Tm}(\mathrm{n}, \gamma){ }^{170} \mathrm{Tm}$ reaction at the ILL using the GAMS 1,2/3 and BIL spectrometers; energies and intensities of primary gamma rays from average resonance capture (ARC, 2- and 24-keV neutron beams) from measurements at the BNL High Flux Beam Reactor; energies and intensities of protons from measurements of the ${ }^{169} \mathrm{Tm}(\mathrm{d}, \mathrm{p})^{170} \mathrm{Tm}$ reaction at the TUM tandem accelerator.

The already existing knowledge of the decay scheme of $170 \mathrm{Tm}$ formed the basis for adding new excited levels in our investigation. ${ }^{2}$ The rotational bands at energies $\left(\mathrm{K}^{\pi}\right.$ of 0 $\mathrm{keV}\left(1^{-}\right), 149.7 \mathrm{keV}\left(0^{-}\right)$, and $204.5 \mathrm{keV}\left(2^{-}\right)$were most instrumental in this regard. We determined additional level energies from our low-energy gamma-ray data by use of the Ritz combination principle, measured multipolarities, and intensity considerations. With few exceptions, the evidence for a level in $170 \mathrm{Tm}$ includes some independent indication of its existence, in addition to the group of secondary gamma transitions that make up the Ritz combination. The independent determinations include primary gamma transitions from thermal neutron capture and/or ARC and proton or alpha groups that appear in the $(\mathrm{d}, \mathrm{p})$ or $(t, \alpha)$ spectra.

Thus, a decay scheme comprised of 48 levels and 149 secondary transitions has been established. These levels have been arranged in rotational bands with the aid of a semi empirical model that employs experimental data on quasiparticle excitations and rotational parameters from neighboring nuclei. ${ }^{3}$ These 17 bands are listed in Table 2, along with their two-quasiparticle configuration assignments. The empirical band structure in ${ }^{170} \mathrm{Tm}$ corresponds moderately well with that predicted by our semi-empirical model. The mean deviations between model and experiment are $81 \mathrm{keV}$ for bandhead energies and $10.4 \%$ for rotational parameters. The experimental and calculated $n-p$ interaction matrix elements show good agreement, with the exception of the $\mathrm{E}(\mathrm{GM})$ matrix element for the $\mathrm{AZ}$ configuration.

\section{Gallagher-Moszkowski Matrix Elements}

The n-p interaction between unpaired nucleons in odd-odd deformed nuclei becomes manifest in two kinds of observables: 1) the Gallagher-Moszkowski energy splitting of rotational bands with identical Nilsson quantum numbers, but with opposite relative orientation of the projections of odd-nucleon angular momentum, and 2) the Newby shift, which is exhibited by $\mathrm{K}=0$ bands wherein the levels with odd angular 
Table 2. Experimental and calculated band energies in ${ }^{170} \mathrm{Tm}$

\begin{tabular}{|c|c|c|c|c|c|c|c|c|}
\hline \multicolumn{3}{|c|}{ BANDHEAD ENERGIES } & \multirow{2}{*}{$\begin{array}{l}\text { \#Lvl } \\
\text { Obs }\end{array}$} & \multicolumn{3}{|c|}{ ROTATIONAL PARAMETERS } & \multicolumn{2}{|c|}{$\mathrm{E}(\mathrm{GM})$ AND E(N) } \\
\hline Config. ${ }^{\mathrm{a}}$ & $\begin{array}{l}\text { Exp. } \\
(\mathrm{keV})\end{array}$ & $\begin{array}{l}\text { Calc.b } \\
(\mathrm{keV})\end{array}$ & & $\begin{array}{l}\text { Exper } \\
\quad(\mathrm{ke}\end{array}$ & $\begin{array}{l}\text { imentalc } \\
\text { V) }\end{array}$ & $\begin{array}{l}\text { Calc.b } \\
\text { (keV) }\end{array}$ & $\begin{array}{c}\text { Exp. } \\
(\mathrm{keV})\end{array}$ & $\begin{array}{l}\text { Calc. } \\
(\mathrm{keV})\end{array}$ \\
\hline $1^{-} \mathrm{AZ}$ & 0.000 & 0 & 7 & $\begin{array}{l}11.454(1,3) \\
11.377(3,5) \\
11.43(2) 5.7\end{array}$ & $\begin{array}{l}10.347(2,4) \\
10.27(3) 4,6\end{array}$ & $10.9(7)$ & +192 & +92 \\
\hline $0-\mathrm{AZ}$ & $\begin{array}{r}149.718(1) \\
(\mathrm{I}=0)\end{array}$ & 52 & 6 & $\begin{array}{l}11.665(0,2) \\
11.551(2,4)\end{array}$ & $\begin{array}{l}11.249(1,3) \\
11.167(3,5)\end{array}$ & $10.9(7)$ & $E(N)=+32$ & +31 \\
\hline $3^{+} \mathrm{AX}$ & $183.193(1)$ & $137(74)$ & 4 & $7.994(3,4)$ & $7.99(7) 4,5$ & $7.3(2)$ & -164 & -144 \\
\hline $4^{+} A X$ & $355.048(1)$ & $283(74)$ & 1 & -. & & $7.3(2)$ & & \\
\hline 2-AY & $204.449(1)$ & $22(15)$ & 4 & $\begin{array}{l}11.016(2,3) \\
10.974(4,5)\end{array}$ & $10.946(3,4)$ & $11.2(3)$ & -231 & -237 \\
\hline 3-AY & $447.070(1)$ & $271(15)$ & 3 & $11.582(3,4)$ & & $11.2(3)$ & & \\
\hline $3+B Z$ & $402.728(1)$ & $352(23)$ & 1 & - - & & $9.5(4)$ & & \\
\hline 1 -EZ & $590.232(1)$ & $598(53)$ & 5 & $\begin{array}{l}11.918(1,2) \\
11.6(2) 4,5\end{array}$ & $11.937(2,4)$ & $11.1(5)$ & & \\
\hline $1+D Z$ & $603.986(1)$ & $583(183)$ & 2 & $11.596(1,2)$ & & $8.1(19)$ & +44 & +49 \\
\hline $0^{+} \mathrm{DZ}$ & $\begin{array}{r}661.854(1) \\
(\mathrm{I}=1)\end{array}$ & $606(183)$ & 2 & -. & & $8.1(19)$ & -. & $g(N)=-34$ \\
\hline $1-\mathrm{AV}$ & $648.745(1)$ & 753(94) & 3 & $10.110(1,3)$ & & $10.8(14)$ & -195 & -180 \\
\hline 2-AV & $854.337(2)$ & $944(94)$ & 2 & $11.824(2,3)$ & & $10.8(14)$ & & \\
\hline $0-\mathrm{BX}$ & $\begin{array}{r}683.567(2) \\
(I=0)\end{array}$ & $567(97)$ & 3 & $8.374(0,2)$ & & $6.6(2)$ & $E(N)=+41$ & +31 \\
\hline 3-DX & $715.619(1)$ & $727(257)$ & 2 & $.5 .074(3,4)$ & & $5.9(10)$ & & \\
\hline $0^{+} \mathrm{CX}$ & $\begin{array}{r}719.264(1) \\
(I=1)\end{array}$ & $576(234)$ & 1 & -. & & $7.5(4)$ & & \\
\hline $2+D Y$ & $758.327(1)$ & 694(198) & 1 & $\cdots$ & & $8.2(18)$ & & \\
\hline 1-EY & $863.369(3)$ & $801(68)$ & 2 & $11.269(1,2)$ & & $11.4(2)$ & & \\
\hline
\end{tabular}

Protons: $\mathrm{A}=1 / 2^{+}[411], \mathrm{B}=7 / 2-[523], \mathrm{C}=7 / 2^{+}[404], \mathrm{D}=1 / 2-[541], \mathrm{E}=3 / 2^{+}[411]$

Neutrons: $Z=1 / 2-[521], Y=5 / 2-[512], X=7 / 2^{+}[633], W=1 / 2-[510], V=3 / 2-[521]$ 
Table 2, footnotes:

a) Nilsson quantum numbers for the unpaired nucleons are designated by capital letters according to the scheme shown at the end of the table.

b) Numbers given in parentheses represent for the indicated quantity the spread in calculated values that arises from taking experimental excitation energies or rotational parameters from two neighboring odd-mass nuclei.

c) Two notations are used in this column: 1) If the value of the rotational parameter is quoted to the nearest electron volt, the experimental uncertainty is $\leq 1 \mathrm{eV}$ and the two numbers in parenthesis are the values of I for the two levels involved, or 2) if the value of the rotational parameter is quoted to the nearest hundredth or tenth of a kilovolt, the experimental uncertainty is given in parenthesis and the level spins follow the parentheses.

d) Calculated G-M matrix elements are taken from the CPTL calculations of Ref. 4. Calculated Newby shifts are taken from H. Frisk, Z. Phys. A, 330 (1988) 241.

momenta are systematically shifted in energy from those with even angular momenta. The matrix elements for these energy splittings and energy shifts can be expressed, as follows:

$$
\mathrm{E}(\mathrm{GM})=\left\langle\chi_{\mathrm{p}}^{\mathrm{k}} \chi_{\mathrm{n}}^{-\mathrm{k}}\left|v_{\mathrm{np}}\right| \chi_{\mathrm{n}}^{\mathrm{k}} \chi_{\mathrm{n}}^{-\mathrm{k}}\right\rangle-\left\langle\chi_{\mathrm{p}}^{\mathrm{k}} \chi_{n}^{\mathrm{k}}\left|v_{\mathrm{np}}\right| \chi_{\mathrm{n}}^{\mathrm{k}} \chi_{\mathrm{n}}^{\mathrm{k}}\right\rangle
$$

and

$$
E(N)=\left\langle\chi_{p}^{k} \chi_{n}^{-k}\left|v_{n p}\right| x_{p}^{-k} \chi_{n}^{k}\right\rangle
$$

Here $\chi^{\mathrm{k}}$,s are the intrinsic single-particle wave functions (not explicit in this notation is that $k$ may take different values for the proton and the neutron) and $V_{n p}$ denotes an effective $n-p$ interaction. Both of these phenomena result in energy shifts that are readily observable. Experimental values of $\mathrm{E}(\mathrm{GM})$ matrix elements in the rare earth and actinide regions generally range from 50 to $250 \mathrm{keV}$, with an overall average of approximately $125 \mathrm{keV}$, while $\mathrm{E}(\mathrm{N})$ matrix elements range in absolute value from $3 \mathrm{keV}$ to $154 \mathrm{keV}$, with an overall average value of $35 \mathrm{keV}$. Although modern instrumentation allows the determination of gamma-ray energies and level energies with precision of one to a few eV, the n-p interaction matrix elements must be extracted from the empirical data by making corrections for a variety of other factors that can influence level energy. Thus, it is the uncertainties in these corrections that ultimately determine how accurately we can deduce values for the $n-p$ interaction matrix elements. 
Corrections for the intrinsic rotational energy of bandheads are made using the following expression:

$$
\mathrm{E}_{\mathrm{rot}}=\hbar^{2} / 2 \mathrm{~J}\left[\mathrm{I}(\mathrm{I}+1)-\mathrm{K}^{2}\right]
$$

Another common correction is that of allowing for energy shifts due to Coriolis mixing with rotational bands where $\Delta \mathrm{K}=1$. Particle-particle couplings can also perturb rotational band energies. 4 The unpaired nucleons can couple with the vibrations of the even-even core via the quasiparticle-phonon interaction. Afanasiev et al..$^{5}$ have described the proper theoretical treatment for these vibrational-rotational excitations. The interaction has been taken into account explicitly in recent calculations by Kvasil et al. ${ }^{6}$ They calculated the microscopic structure of intrinsic states in five odd-odd holmium isotopes using the quasiparticle-phonon model originally formulated for odd-mass nuclei by Soloviev 7 , with the inclusion of the $n-p$ interaction. For ${ }^{166} \mathrm{Ho}$, the Ho isotope best understood experimentally, the calculations reproduce the empirical data rather well. As might be expected, the main strength of the gamma vibrations is concentrated in intrinsic states with energies greater than $1 \mathrm{MeV}$, with one exception. A pair of rotational bands with appreciable vibrational components (as much as $26 \%$ ) and $\mathrm{K}^{\pi}=5^{-}$and $2^{-}$are predicted to occur at $260 \mathrm{keV}$ and $525 \mathrm{keV}$, respectively. Experimental evidence for occurrence of a 2band at $543 \mathrm{keV}$ with vibrational character has been reported.$^{8}$ It is evident that the best understanding of level structure in these odd-odd deformed nuclei will come by comparing empirical data with fully microscopic calculations such as those made by Kvasil et al. Until the results of microscopic calculations are available for a wider range of nuclei, much information regarding the $n-p$ interaction matrix elements can be obtained by making simple corrections to band energies for the effects already discussed, particularly if the corrections lead to relatively minor revisions in these energies.

In the earliest theoretical treatments of the n-p interaction, a central force with zero range (delta force) was assumed. ${ }^{9-12}$ The calculated G-M matrix elements showed some correlation with empirical data, but not with very great precision. For example, best fits to modern data sets yield root-mean-squared (ms) deviations of $40-50 \mathrm{keV}$, while the mean value of the matrix elements is approximately $125 \mathrm{keV}$. While attempting to fit data for both the G-M and the Newby matrix elements with a single description of the n-p force, Jones et al. 13 chose to employ a central force of finite range plus a tensor force with an assumed Gaussian radial dependence for both. With this force, they produced a fit with rms deviation of $57 \mathrm{keV}$ to a data set, including $27 \mathrm{G}-\mathrm{M}$ splittings and $7 \mathrm{Newby}$ shifts. It had already been recognized that in the asymptotic limit of large nuclear deformation, a tensor force is required in order to produce the scattering necessary for a Newby shift in a triplet configuration. 9 Bennour et al. ${ }^{14}$ modeled the levels of odd-odd nuclei using the Hartree-Fock approximation. In their description of nuclear states in a deformed nucleus, the single-particle wave functions and energies, the static equilibrium core properties, and the residual $n-p$ interaction are all determined from the same effective interaction (Skyrme III). Their calculated G-M matrix elements, when compared with nineteen experiniental values taken from actinide nuclei, produced an rms deviation of $71 \mathrm{keV}$. 
Their calculated G-M matrix elements, when compared with nineteer experimental values taken from actinide nuclei, produced an rms deviation of $71 \mathrm{keV}$.

The most comprehensive treatment of the experimental and theoretical aspects of the residual $n-p$ interaction is that of Boisson et al. ${ }^{4}$ In addition to testing several assumptions regarding the nature of the $n-p$ force, these authors also tested the use of both modified harmonic-oscillator wave functions and those derived with a Woods-Saxon potential. They found little difference from the use of either form of the wave functions. While several authors have chosen to model the $n-p$ force with a central force of finite range, they produced only a modest improvement in the agreement with G-M matrix element data (rms deviations of approximately $40 \mathrm{keV}$ ), as compared with zero-range force calculations, and at the cost of increasing the number of adjustable parameters from one to four. Boisson et al. produced a very significant improvement in the rms deviation for their G-M matrixelement data set, reducing it to $17 \mathrm{keV}$, by including several additional features in a comprehensive description of the $n-p$ interaction. These included allowing for core polarization by polarizing the intrinsic-spin operators and extending the description of the force by adding both long-range and tensor components, all of which required seven adjustable parameters. These data are listed as items 1-27 in Table 3; the calculated values of Boisson et al. are designated "CPTL". The ratios of experimental G-M matrix elements to CPTL calculations are shown in the left side of Fig. 1. The data show rather good clustering around a ratio of 1.0 ; all but a few of the points lie within the $\pm 20 \%$ lines indicated in the figure.

Since the publication of the Boisson et al. paper in 1976, the body of E(GM) data has grown considerably. For the rare earth region, these data appear as items $29 \mathrm{~b}-51$ in Table 3; the corresponding E(GM) ratios, experimental/calculated, appear in Fig. 1. For those matrix elements measured in ${ }^{152} \mathrm{Eu}$ and ${ }^{154} \mathrm{Eu}, \mathrm{E}(\mathrm{GM})$ values are included in Table 3 and Fig. 1 only for those band-pairs rated as most reliable by the authors. 15,16 It is evident from Fig. 1 that the newer empirical data are not well reproduced by the CPTL calculations; more of the data lie outside of the $\pm 20 \%$ region than lie inside. Thus, the predictive power of the calculations has proven to be relatively poor. This leads one to question the importance of some features of the CPTL force.

We have taken a somewhat different philosophical approach to this problem by examining G-M matrix-element systematics in hopes of finding clues to a better description of the interaction. We have calculated G-M matrix elements by reverting to the assumption of a zero-range force with its one adjustable parameter, these results are listed in column 10 of Table 3 as "Case E". Parameter adjustment was done somewhat arbitrarily so that a majority of the data ratios average to 1.0 ; a least-squares minimum fit to all of the data was not performed. These data ratios have been found to correlate with the factor $x=\left(\Delta n_{z}+\Delta \Lambda\right)$, the sum of the differences in the Nilsson quantum numbers $n_{z}$ and $\Lambda$ for the proton and neutron orbitals, as shown in Fig. 2. For values of $x \geq 2$, the data ratios fall mostly within the $\pm 20 \%$ lines centered about 1.0 . When $x<2$, most of the data ratios exhibit values well 
Table 3. Gallagher-Moszkowski Matrix Elements, Rare Earth Region

Experimental Calculated Ratios

\begin{tabular}{|c|c|c|c|c|c|c|c|c|c|c|c|}
\hline & $\begin{array}{l}\text { Proton } \\
\text { (1) }\end{array}$ & $\begin{array}{l}\text { Neutron } \\
\text { (2) }\end{array}$ & $\begin{array}{l}\text { Nuclide } \\
\text { (3) }\end{array}$ & $\begin{array}{l}\text { Orbitals ( } \\
\text { (4) }\end{array}$ & $\begin{array}{c}n_{2}+\Delta \\
(5)\end{array}$ & $\begin{array}{l}\text { Uncorrected } \\
\text { (6) }\end{array}$ & $\begin{array}{c}\mathrm{BPO} \\
(7)\end{array}$ & $\begin{array}{c}\text { CPTL } \\
(8)\end{array}$ & $\begin{array}{c}\text { Case E } \\
(9)\end{array}$ & $\begin{array}{l}\text { Exp/CPTL } \\
(10)\end{array}$ & $\begin{array}{c}\text { Exp/Cse } \\
\text { (11) }\end{array}$ \\
\hline 1 & $3 / 2+411 \uparrow$ & $3 / 2-521 \uparrow$ & $158,160 \mathrm{~Tb}$ & $\mathrm{~d} 5 \mathrm{f} 7$ & 1 & 128 & 130 & 116 & 202 & 1.12 & 0.63 \\
\hline 2 & & $5 / 2-523 \downarrow$ & ${ }^{160} \mathrm{~Tb}$ & d5h9 & 3 & -165 & -161 & -160 & -138 & 1.01 & 1.20 \\
\hline 3 & & $5 / 2+642 \uparrow$ & ${ }^{160} \mathrm{~Tb}$ & d5i13 & 4 & 93 & 93 & 82 & 97 & 1.13 & 0.96 \\
\hline 4 & $7 / 2-523 \uparrow$ & $1 / 2+400 \uparrow$ & ${ }^{164} \mathrm{Ho}$ & h11s1 & 5 & 101 & 101 & 77 & 107 & 1.31 & 0.94 \\
\hline 5 & & $3 / 2+402 \downarrow$ & ${ }^{164} \mathrm{Ho}$ & h11d3 & 3 & -88 & -88 & -76 & -126 & 1.16 & 0.70 \\
\hline 6 & & $5 / 2-523 \downarrow$ & ${ }^{164 \mathrm{Ho}}$ & h11h9 & 0 & -148 & -148 & -154 & -322 & 0.96 & 0.46 \\
\hline 7 & & $7 / 2+633 \uparrow$ & ${ }^{166} \mathrm{Ho}$ & h11i13 & 1 & 80 & 84 & 101 & 235 & 0.83 & 0.34 \\
\hline 8 & & $1 / 2-521 \downarrow$ & ${ }^{166} \mathrm{Ho}$ & h11f5 & 2 & -172 & -167 & -159 & -169 & 1.05 & 1.02 \\
\hline 9 & $1 / 2+411 \downarrow$ & $7 / 2+633 \uparrow$ & ${ }^{168} \mathrm{Tm}$ & $\mathrm{d} 3 \mathrm{i} 13$ & 4 & -151 & -138 & -144 & -148 & 0.96 & 1.02 \\
\hline 10 & & $5 / 2-512 \uparrow$ & ${ }^{170} \mathrm{Tm}$ & $13 \mathrm{f} 7$ & 1 & -231 & -232 & -237 & -330 & 0.98 & 0.70 \\
\hline 11 & $1 / 2-541 \downarrow$ & $7 / 2+633 \uparrow$ & ${ }^{168} \mathrm{Tm}$ & h9i13 & 3 & -76 & -76 & -77 & & 0.99 & \\
\hline 12 & & $5 / 2-512 \uparrow$ & ${ }^{174} \mathrm{Lu}$ & h9f7 & 4 & -7 & -25 & -26 & & 0.96 & \\
\hline 13 & $7 / 2+404 \downarrow$ & $1 / 2-521 \downarrow$ & ${ }^{174} \mathrm{Lu}$ & g7f5 & 5 & 80 & 75 & 90 & 80 & 0.83 & 1.00 \\
\hline 14 & & $3 / 2-521 \uparrow$ & ${ }^{174} \mathrm{Lu}$ & g7f7 & 5 & -99 & -99 & -83 & & 1.19 & \\
\hline 15 & & $5 / 2-512 \uparrow$ & ${ }^{174} \mathrm{Lu}$ & $\mathrm{g} 7 \mathrm{f} 7$ & 3 & -118 & -114 & -109 & -127 & 1.05 & 0.93 \\
\hline 16 & & $7 / 2+633 \uparrow$ & ${ }^{174} \mathrm{Lu}$ & g7i13 & 4 & -111 & -90 & -75 & -152 & 1.20 & 0.73 \\
\hline 17 & & $9 / 2+624 \uparrow$ & ${ }^{176} \mathrm{Lu}$ & g7i13 & 2 & -130 & -130 & -107 & & 1.21 & \\
\hline 18 & & $7 / 2514 \downarrow$ & ${ }^{176} \mathrm{Lu}$ & g7h9 & 1 & 249 & 253 & 219 & 257 & 1.16 & 0.97 \\
\hline 19 & & $1 / 2-510 \uparrow$ & ${ }^{176} \mathrm{Lu},{ }^{182} \mathrm{Ta}$ & g7p3 & 5 & -102 & -100 & -109 & -123 & 0.92 & 0.83 \\
\hline 20 & & $3 / 2-512 \downarrow$ & ${ }^{182} \mathrm{Ta}$ & g7f5 & 3 & 144 & 89 & 126 & 132 & 0.71 & 1.09 \\
\hline 21 & & $7 / 2-503 \uparrow$ & ${ }^{182} \mathrm{Ta}$ & g7f7 & 1 & -123 & -123 & -153 & -293 & 0.80 & 0.42 \\
\hline 22 & $1 / 2-530 \uparrow$ & $5 / 2-512 \uparrow$ & ${ }^{174} \mathrm{Lu}$ & f7f? & 4 & 31 & 31 & 56 & & 0.55 & \\
\hline 23 & $9 / 2-514 \uparrow$ & $5 / 2-512 \uparrow$ & ${ }^{174} \mathrm{Lu}$ & h11f7 & 2 & 168 & 168 & 160 & 218 & 1.05 & 0.77 \\
\hline 24 & & $1 / 2-510 \uparrow$ & ${ }^{182} \mathrm{Ta}$ & h11p3 & 4 & 159 & 159 & 150 & 194 & 1.06 & 0.82 \\
\hline 25 & $5 / 2+402 \uparrow$ & $1 / 2-510 \uparrow$ & $186,188 \mathrm{Re}$ & $\mathrm{d} 5 \mathrm{p} 3$ & 3 & 112 & 102 & 115 & 119 & 0.89 & 0.94 \\
\hline 26 & & $3 / 2-512 \downarrow$ & $186,188 \mathrm{Re}$ & $\mathrm{d} 5 \mathrm{f} 5$ & 1 & -156 & -156 & -166 & -214 & 0.94 & 0.73 \\
\hline 27 & & $7 / 2-503 \uparrow$ & $186,188 \mathrm{Re}$ & $\mathrm{d} 5 \mathrm{f} 7$ & 1 & 209 & 208 & 213 & 486 & 0.98 & 0.43 \\
\hline
\end{tabular}


Table 3. GM Matrix Elements, Rare Earth Region (continued)

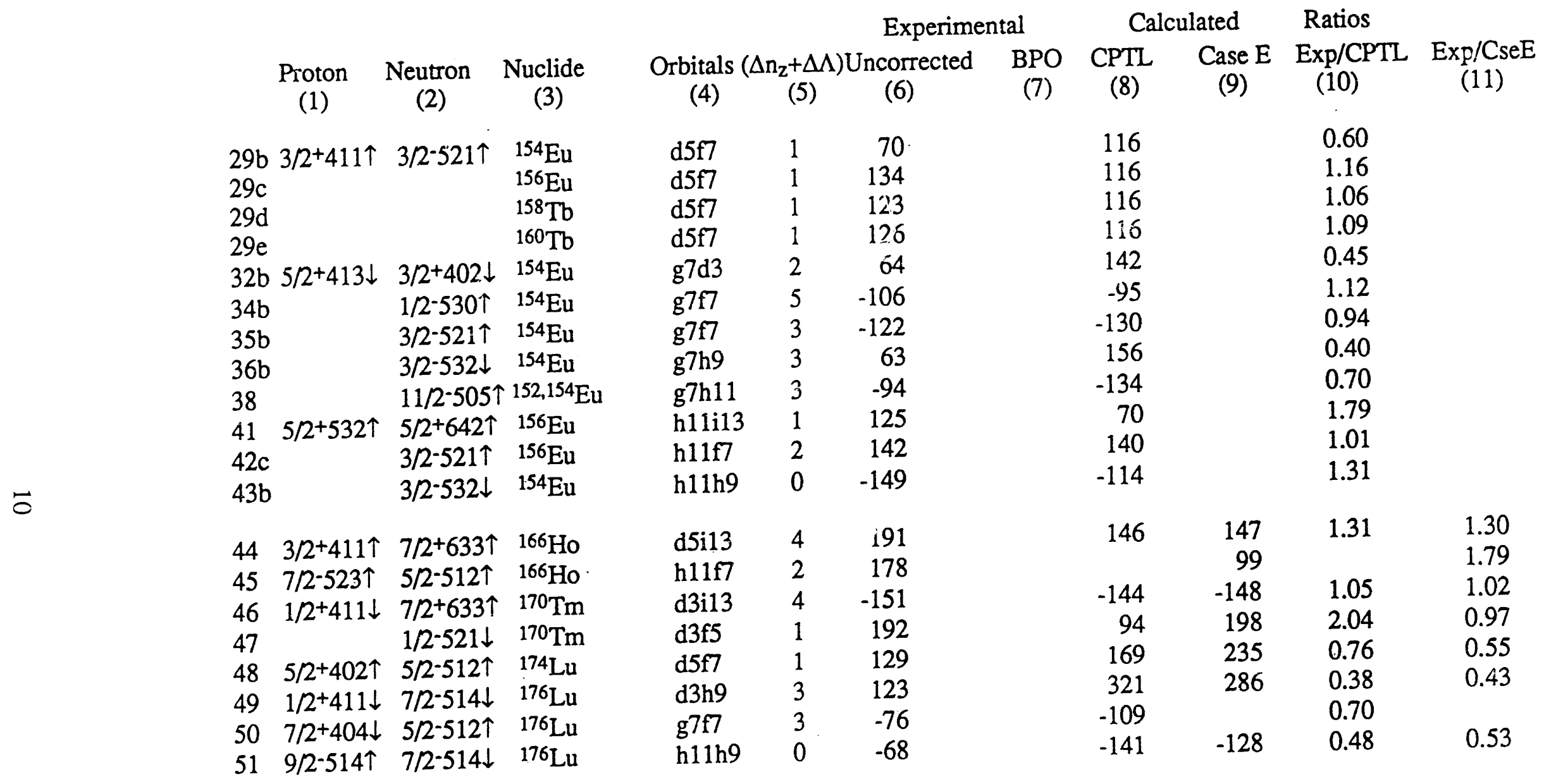


below 1.0, with two exceptions. Thus, it appears that when $x=0$ or 1 the theoretical expression for $\mathrm{E}(\mathrm{GM})$ tends to produce values that are usually too large, i.e. the amount of proton and neutron wave function overlap is overestimated. Some sort of damping factor seems to be operating to reduce the effective interaction between the odd nucleons when their wave functions are quite similar, whereas the theoretical expression tends to produce a realistic amount of overlap when the wave functions are more dissimilar.

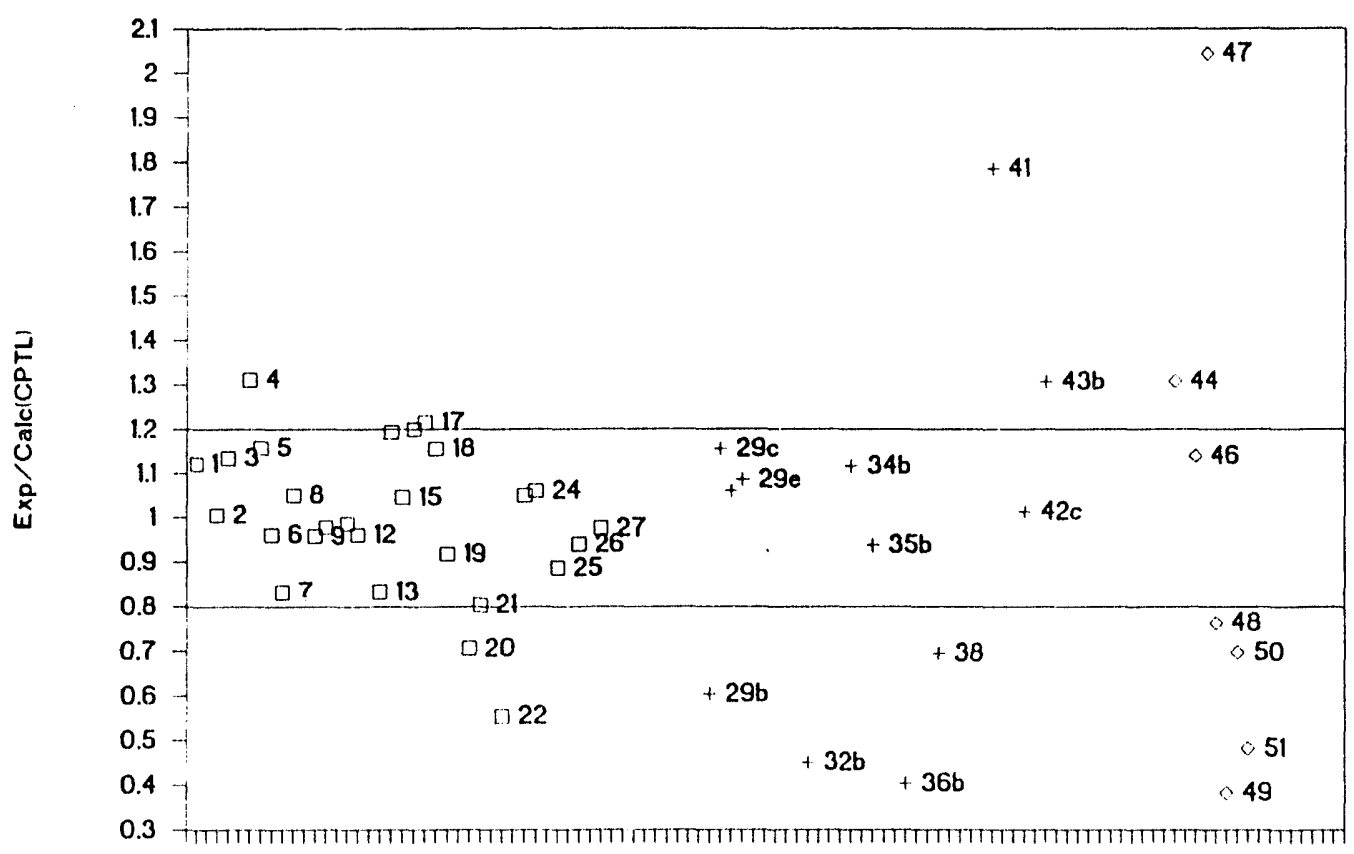

[1 BPO data + Eus o New

Figure 1. Ratios of experimental to calculated values for Gallagher-Moszkowski matrix elements from odd-odd nuclei in the rare earth region. The data are listed in column 10 of Table 3.

It is clear in Fig. 2 that a few of the data points in the region $x \geq 2$ lie far from a ratio of 1.0. It should be noted that none of the data have been corrected for various energy shifts, as discussed in an earlier paragraph, except that all have been corrected for the intrinsic rotational energy of the bandhead level. More rigorous treatment of these corrections or even a full microscopic calculation of level structure may result in an improved clustering of the data where $x \geq 2$. On the other hand, corrections that will bring the most extreme outliers into agreement seem to be much larger than our estimates of these effects. 
The data for $\mathrm{x}=1$ also exhibit an interesting correlation. For those data where the proton is in a d orbital and the neutron is in a $\mathrm{f}$ orbital, there is a regular decrease in the data ratio as $\Sigma j$ increases from a value of 4 to 6 .

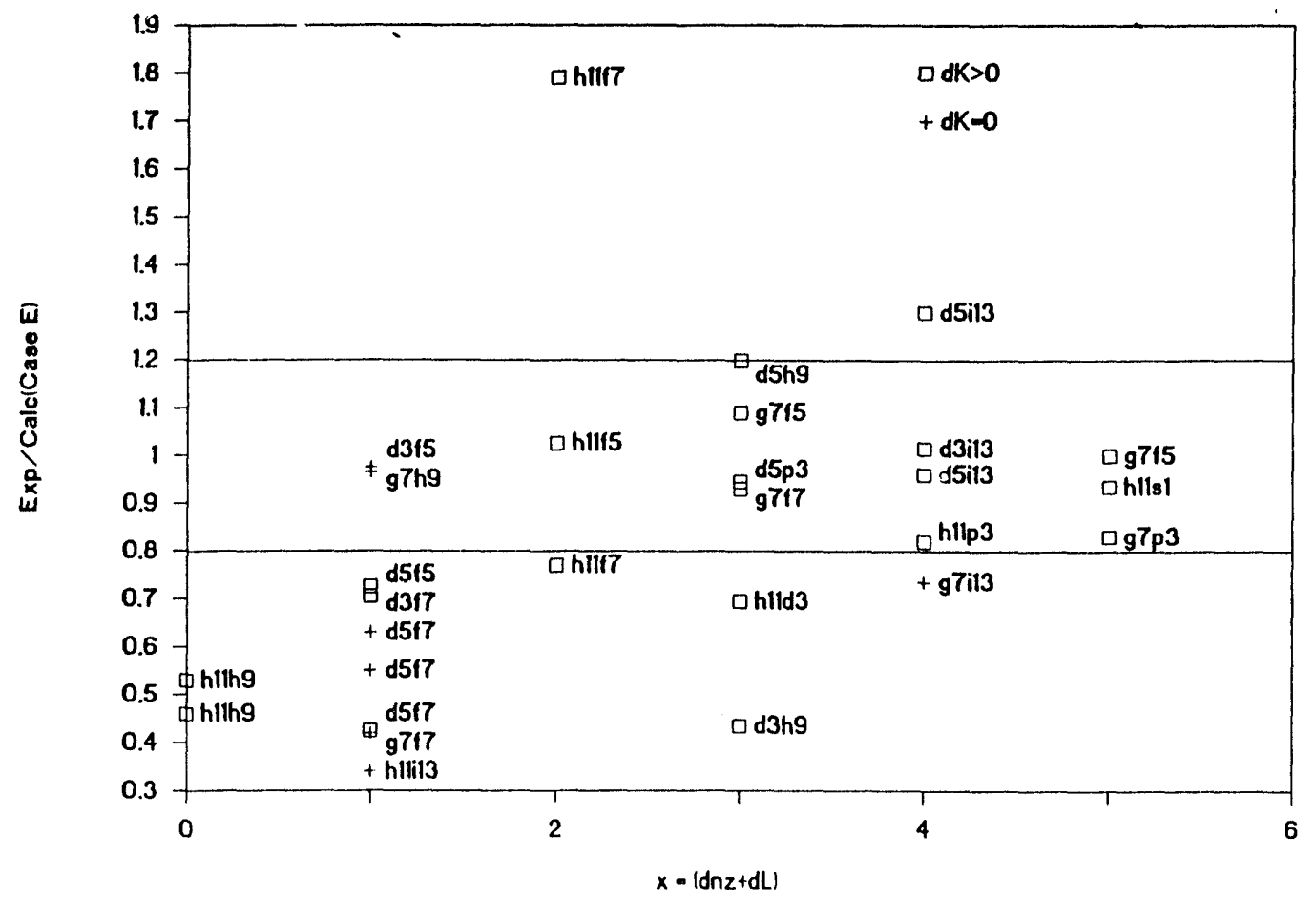

Figure 2. Systematic behavior of Gallagher-Moszkowski data ratios with the parameter $x=\left(\Delta n_{z}+\Delta \Lambda\right)$. The data are labeled according to the shell models for the unpaired proton and neutron (each number equals 2j). The data are listed in columns 5 and 11 of Table 3.

Certain of the experimental data in Table 3 are not included in the correlation shown in Fig. 2. Calculations have not yet been performed for the europium isotopes. Calculated matrix elements for configurations including a 1/2541 proton were excluded because they were found to be very sensitive to parameters specifying nuclear shape and the Nilsson potential. For others, numbers 14,17 , and 22 in Table 3 , there were questions regarding the reliability of the configuration assignments. A similar trend to that shown in Fig. 2 is found for $\mathrm{E}(\mathrm{GM})$ 's in the odd-odd actinide nuclei.

We propose the rare earth region G-M matrix elements can be understood as following calculations that assume a zero-range force when $x=\left(\Delta n_{z}+\Delta \Lambda\right)$ is $\geq 2$ and tending to be damped, exhibiting less overlap than calculated, when $\mathrm{x}<2$. The origin of this behavior is not yet understood. 


\section{Acknowledgments}

It is a pleasure to thank the following collaborators for their part in the experimental study of ${ }^{170} \mathrm{Tm}$ transitions and level structure: At the Institut Laue-Langevin, Grenoble, H.G. Boerner, K. Schreckenbach, G.G. Colvin, and F. Hoyler; at the Technical University of Munich, T. von Egidy, W. Schauer, S. Schruender, and R. Georgii, ; at Brookhaven National Laboratory, R.F. Casten and R. Gill; at the Bruyeres-le-Chatel Laboratory, S. Joly and O. Bersillon. I am grateful to the following persons for helpful discussions and collaboration in the study of G-M matrix elements: at Banaras Hindu University, India, P.C. Sood; at the Florida State University, R.K. Sheline; at Charles University, Prague, J. Kvasil; at the University of Roorkee, India, A.K. Jain.

\section{References}

* Work performed under the auspices of the U.S. Department of Energy by the Lawrence Livermore National Laboratory under contract W-7405-ENG-48.

1. P. C. Sood, D. M. Headly, R. K. Sheline, and R. W. Hoff, to be published in At. Data and Nucl. Data Tables (1993).

2. Z. Chunmei, Nucl. Data Sheets 50 (1987) 225.

3. R. W. Hoff, J. Kern, R. Piepenbring, and J. P. Boisson, Proc. Fifth Int'l. Symp. on Capture Gamma-Ray Spectroscopy and Related Topics, Conf. Proc. No. 125, ed. S. Raman (AIP, New York 1985) 274; T. von Egidy et al., Phys. Rev. C29 (1984) 1243.

4. J. P. Boisson, R. Piepenbring, and W. Ogle, Phys. Rpts. 26 (1976) 99.

5. A. V. Afanasiev, T. V. Guseva, and J. J. Tambergs, Bull. Acad. Sci. USSR, Phys. Ser. 52 (1988) 121; ibid. 53 (1989) 52.

6. J. Kvasil, R. K. Sheline, V. O. Nesterenko, I. Hrivnacova, and D. Nosek, Z. Phys. A 343 (1992) 145.

7. V. G. Soloviev, Phys. Lett. 21 (1966) 320.

8. M. K. Balodis, A. V. Afanasiev, P. T. Prokofiev, Yu. Ya. Tambergs, Bull. Acad. Sci. USSR, Phys. Ser. 52 (1988) 35.

9. N. D. Newby, Phys. Rev. 125 (1962) 2063. 
10. N. I. Pyatov, Bull. Acad. Sci. USSR, Phys. Ser. 27 (1963) 1409; N. I. Pyatov and A. S. Chernyshev, Bull. Acad. Sci. USSR, Phys. Ser. 28 (1964) 1073.

11. L. A. Neiburg, P. T. Prokofjevs, and J. J. Tambergs, Bull. Acad. Sci. USSR, Phys. Ser. 36 (1972) 2220.

12. P. C. Sood and R. N. Singh, Nucl. Phys. A373 (1982) 519; Phys. Rev. C25 (1982) 1632; P. C. Sood, R. K. Sheline, a.1d R. S. Ray, Phys. Rev. C35 (1987) 1922; P. C. Sood, R. K. Sheline, and R. W. Hoff, Pramana 30 (1988) 93.

13. H. D. Jones, N. Onishi, T. Hess, and R. K. Sheline, Phys. Rev. C3 (1971) 529.

14. L. Bennour, J. Libert, M. Meyer, and P. Quentin, Nucl. Phys. A465 (1987) 35

15. T. von Egidy et al., Z. Phys. A286 (1978) 341.

16. M. K. Balodis et al., Nucl. Phys. A472 (1987) 445. 

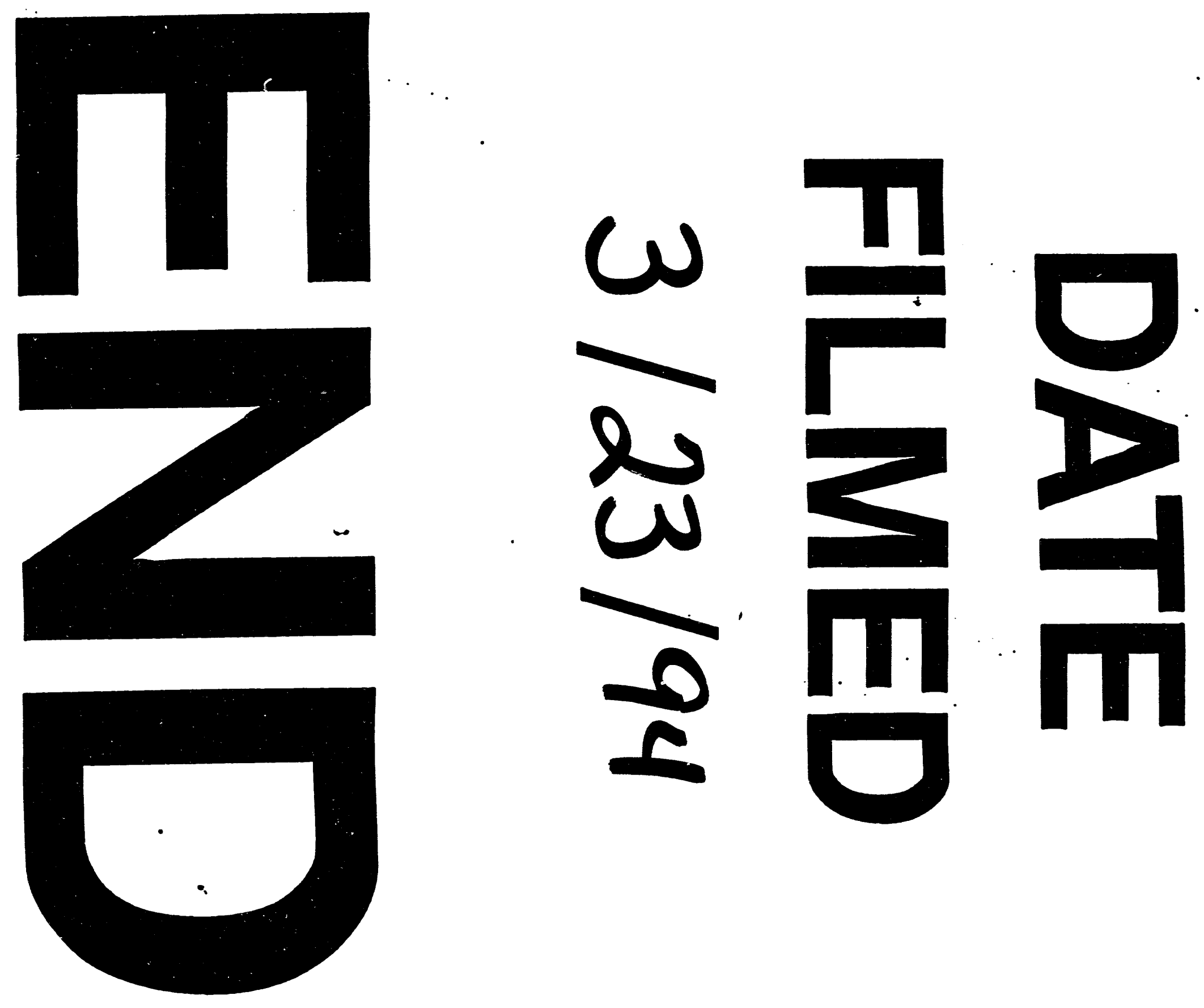


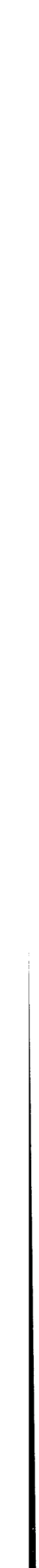

